\title{
EDITORIAL
}

\section{Could resuscitation be based on microcirculation data? We are not sure}

Xavier Monnet ${ }^{1 *}$ and Bernd Saugel ${ }^{2}$

(c) 2018 Springer-Verlag GmbH Germany, part of Springer Nature and ESICM

\section{Introduction}

Malpighi described the connections between the arteries and veins in 1661 by observing the capillaries under the microscope. In intensive care, though the physiological importance of microcirculation has been known for a long time [1], an important step was taken in the late 1990s, when a technology borrowed from intraoperative neurosurgical exploration allowed direct visualisation of the microvessels in vivo. Since then, a considerable number of experimental and clinical studies have been carried out in this field.

Today, we have come to a debate between those who believe that microcirculation monitoring should be a part of shock management and those who think it is scientific "l'art pour l'art" without consequences for clinical decision-making. Some claim that microcirculatory alterations play a pivotal role in the pathophysiology of shock and that available techniques to explore the microcirculation should be used to guide therapeutic choices. Others argue that the available techniques are expensive, fraught with limitations and that no treatment can be guided by their use, especially because consensus on the subject is lacking.

What should we think? It seems to us that for haemodynamic resuscitation to be guided by microcirculatory data, three conditions should be fulfilled. First, it should be established that microcirculatory failure is of paramount importance in the development, course and prognosis of shock states. Secondly, techniques exploring

\footnotetext{
*Correspondence: xavier.monnet@aphp.fr

${ }^{1}$ Medical Intensive Care Unit, Bicêtre Hospital, Paris-Sud University

Hospitals, 78, rue du Général Leclerc, Le Kremlin-Bicêtre 94270, France
}

Full author information is available at the end of the article

For contrasting viewpoints, please go to https://doi.org/10.1007/s00134018-5095-y and https://doi.org/10.1007/s00134-018-5121-0. microvessels should be efficient and easy to use at the bedside. Thirdly, assessing the microcirculation should lead to therapeutic interventions that would not be decided otherwise. Are these conditions fulfilled today?

\section{Does the microcirculation play a crucial role in the pathophysiology of shock?}

The pathophysiological importance of the microcirculation in shock states has been known for years. In the face of decreased arterial oxygen transport, the increase in oxygen extraction is basically explained by the dilation of microvessels under the effect of substances produced locally, by the cells or by the endothelium [2]. This "metabolic autoregulation", which is one of the body's defences against circulatory failure, has been established for a long time.

It is also well established that this autoregulation malfunctions during septic shock. Decreased sensitivity of microvessels to vasoactive substances, destruction of glycocalyx, rheological blood disturbances and activation of coagulation contribute to microcirculatory failure [3]. Techniques visualising the microcirculation essentially contributed to the understanding of septic microcirculatory impairment [4]. A decrease in the proportion of perfused capillaries, a decrease in blood flow in the capillaries that remain perfused and the formation of underperfused areas in the midst of large arteriovenous shunts characterise septic shock [3]. Such abnormalities have also been observed in a wide range of diseases other than sepsis.

The pathophysiological importance of this microcirculatory failure is reinforced by studies that have shown, following that of De Backer et al. in 2002 [4], that it is more pronounced and that it improves less among sepsis non-survivors than survivors. In fact, there are cases in which microcirculatory failure takes precedence over

\section{Springer}


macrocirculatory failure, where tissue hypoxia persists while cardiac output and blood pressure have been optimised [5]. This "loss of coherence" between micro- and macrocirculation [6] is not systematic. Clinical practice tells us that, in most cases, the correction of macrohaemodynamic disorders reduces tissue hypoxia, as far as can be judged by the biological markers (lactate, carbon dioxide-derived indices) or by the function of organs like the kidneys. Patients who die of haemodynamic failure do not die with normal blood pressure. Nevertheless, it cannot be denied that, in some cases, microcirculatory failure persists to varying degrees, and that specifically correcting this failure should improve the outcome of these patients. Nevertheless, to justify a specific treatment and monitoring of the microcirculation, it is not necessary to demonstrate that this "loss of coherence" exists. The ideal therapeutic strategy of shock should act both on micro- and macrocirculatory disorders.

\section{Are the techniques to explore the microcirculation ready for routine clinical use?}

It is undeniable that the techniques visualising microvessels in vivo have led to considerable advances in the knowledge about their pathophysiology. Today, handheld vital microscopy (HVM) - using either sidestream or incident dark-field imaging-is the technique used mainly. It evaluates the microcirculation anatomy and function through various variables [7]. Near-infrared spectroscopy, which measures the oxygen saturation of haemoglobin in muscle and brain, was interesting in the field of shock as it assessed the microvascular reactivity in response to an ischemic stress. Unfortunately, its commercial development for this use has been stopped. The perfusion index, which is the ratio of the pulsatile to nonpulsatile part of the plethysmography signal, provides an easy and cheap estimation of the skin perfusion. By contrast, transcutaneous oximetry, which measures the skin partial pressure in oxygen, requires Clarke electrodes placed on the skin surface.

Whatever they are, these techniques still have major limitations today. The main one is that they explore only one particular site, whose pathophysiological importance is not necessarily major or representative of other regions. This is obvious for plethysmography and transcutaneous oximetry. Regarding HVM, although it is hypothesised that the sublingual circulation reflects that of the splanchnic territory, it does not respond in the same way as other important vascular territories [8]. Moreover, it is true to say that HVM requires experience in order to avoid many pitfalls. The interposition of saliva between the probe and the mucosa and the excessive pressure force of the probe on this mucosa are factors that one must learn to avoid [7]. Finally, it is true that
HVM requires a long, tedious and complex analysis of the images and that it is expensive. This is not the case for the perfusion index, but the latter is limited by the fact that, in case of strong vasoconstriction, the plethysmography signal may even be impossible to obtain. Moreover, the perfusion index is also influenced by cardiac output and provides much less information than sidestream dark-field imaging (SDF). This is also the case for transcutaneous oximetry.

However, it is a safe bet that these technical limitations will be overcome in the near future. In this field, as in almost every other field of intensive care medicine, the engineers of the technology industry will lead to the progress that is expected. Regarding HVM, many attempts have been made already to automate the analysis of images [9], even though their use in clinical evaluation has yet to be validated [7]. In the future, it is certain that HVM tools will allow easy and rapid exploration of microvessels in the sublingual region but also in other areas.

\section{Does the assessment of the microcirculation trigger specific therapeutic interventions?}

Almost all treatments used in critically ill patients have been explored with regard to their effect on the microcirculation [10], ranging from fluids to extracorporeal support and blood transfusions. The problem is that there is no vasoactive treatment whose microvascular effects are dissociated from the effects on the large vessels, and sometimes on the cardiac function. Often, the beneficial effects on both components are added, as is the case for fluids, dobutamine and levosimendan [10]. In other cases, the macrocirculatory effects of the treatments counteract their macrocirculatory beneficial effect, as it may be the case for norepinephrine, although its effects on the microcirculation vary from one patient to another [11]. The example of nitrates is illustrative. Their hypotensive effect is opposed to the beneficial vasodilation that they could exert on the microcirculation. This effect was well demonstrated during septic shock [12], but in patients whose macrocirculatory function was already restored, and this effect was not confirmed in a randomised controlled trial [13]. Depret and co-workers interestingly reported three cases of septic shock with severe skin vasoconstriction in which iloprost improved local perfusion without altering cardiac output and arterial pressure [14]. Nevertheless, in this study, macrohaemodynamics had been optimised before starting the treatment. It is illusory to think that, in the future, a microcirculationspecific vasodilator may appear since large and small vessels are regulated by the same pathways.

By contrast, the theoretical interest may come from treatments modulating coagulation, the activation of 


\section{Prerequisites for the clinical use of microcirculation monitoring}

\section{Pathophysiology}

The microcirculation plays a crucial role in the development and course of circulatory shock

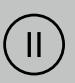

Microcirculation monitoring can be easily performed at the bedside<smiles>[CH]CC</smiles>

artifact-free computed-controlled image acquisition + automatic image analysis

Therapy

Microcirculation monitoring triggers specific therapeutic interventions

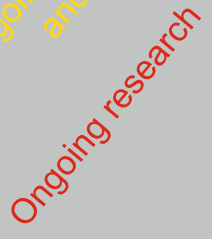

Fig. 1 What are the prerequisites for using microcirculation monitoring at the bedside?

which participates in septic microvascular dysfunction. Clear beneficial effects were described for activated protein $C$ [15], and could be expected from similar drugs in the future. Also, strategies in the future will likely consider the microcirculation in the therapeutic strategy. For example, microcirculation data could guide the choice whether or not to start an inotropic drug, to perform a blood transfusion or when choosing between strengthening vasopressors and infusing fluids. Clinical evidence of such strategies has not been established, but these are interesting leads for the future (Fig. 1).

In conclusion, in view of the considerable pathophysiological importance of the microcirculation during shock states, the development of techniques to explore the microcirculation and the extremely interesting research that they have allowed must be considered as a chance and an opportunity. In the future, it is highly likely that improved monitoring techniques will enable therapeutic choices to be guided by microcirculatory variables in patients with acute circulatory failure. Can resuscitation be guided by microcirculatory data? Today no, but tomorrow, no doubt!

\section{Author details}

${ }^{1}$ Medical Intensive Care Unit, Bicêtre Hospital, Paris-Sud University Hospitals, 78, rue du Général Leclerc, Le Kremlin-Bicêtre 94270, France. ${ }^{2}$ Department of Anesthesiology, Center of Anesthesiology and Intensive Care Medicine, University Medical Center Hamburg-Eppendorf, Hamburg, Germany.

\section{Compliance with ethical standards}

\section{Conflicts of interest}

Xavier Monnet is a member of the medical advisory board of Pulsion Medical Systems (part of Maquet Getinge group). He has received honoraria for giving lectures from Pulsion Medical Systems, Cheetah Medical and Masimo.Bernd Saugel is a member of the medical advisory board of Pulsion Medical Systems (part of Maquet Getinge group). He has received honoraria for giving lectures from Pulsion Medical Systems and CNSystems Medizintechnik AG. He received refunds of travel expenses from Pulsion Medical Systems, Tensys Medical Inc and CNSystems Medizintechnik AG. He received research grants, unrestricted research grants from Tensys Medical Inc and received research support from Edwards Lifesciences.

Received: 7 March 2018 Accepted: 11 April 2018 Published online: 7 May 2018

\section{References}

1. Johnson PC (1972) Renaissance in the microcirculation. Circ Res 31(6):817-823

2. Schumacker PT, Cain SM (1987) The concept of a critical oxygen delivery. Intensive Care Med 13(4):223-229

3. Miranda M, Balarini M, Caixeta D, Bouskela E (2016) Microcirculatory dysfunction in sepsis: pathophysiology, clinical monitoring, and potential therapies. Am J Physiol Heart Circ Physiol. 311(1):H24-35

4. De Backer D, Creteur J, Preiser JC, Dubois MJ, Vincent JL (2002) Microvascular blood flow is altered in patients with sepsis. Am J Respir Crit Care Med 166(1):98-104

5. De Backer D, Donadello K, Sakr Y, Ospina-Tascon G, Salgado D, Scolletta S et al (2013) Microcirculatory alterations in patients with severe sepsis: impact of time of assessment and relationship with outcome. Crit Care Med 41(3):791-799 
6. Ince C (2015) Hemodynamic coherence and the rationale for monitoring the microcirculation. Crit Care 19(Suppl 3):S8

7. Ince C, Boerma EC, Cecconi M, De Backer D, Shapiro NI, Duranteau J et al (2018) Second consensus on the assessment of sublingual microcirculation in critically ill patients: results from a task force of the European Society of Intensive Care Medicine. Intensive Care Med. 44(3):281-299

8. Edul VS, Ince C, Navarro N, Previgliano L, Risso-Vazquez A, Rubatto PN et al (2014) Dissociation between sublingual and gut microcirculation in the response to a fluid challenge in postoperative patients with abdominal sepsis. Ann Intensive Care. 4:39

9. Carsetti A, Aya HD, Pierantozzi S, Bazurro S, Donati A, Rhodes A et al (2017) Ability and efficiency of an automatic analysis software to measure microvascular parameters. J Clin Monit Comput 31 (4):669-676

10. Saugel B, Trepte CJ, Heckel K, Wagner JY, Reuter DA (2015) Hemodynamic management of septic shock: is it time for "individualized goal-directed hemodynamic therapy" and for specifically targeting the microcirculation? Shock 43(6):522-529

11. Dubin A, Pozo MO, Casabella CA, Palizas F Jr, Murias G, Moseinco MC et al (2009) Increasing arterial blood pressure with norepinephrine does not improve microcirculatory blood flow: a prospective study. Crit Care 13(3):R92

12. Spronk PE, Ince C, Gardien MJ, Mathura KR, Oudemans-van Straaten HM, Zandstra DF (2002) Nitroglycerin in septic shock after intravascular volume resuscitation. Lancet 360(9343):1395-1396

13. Boerma EC, Koopmans M, Konijn A, Kaiferova K, Bakker AJ, van Roon EN et al (2010) Effects of nitroglycerin on sublingual microcirculatory blood flow in patients with severe sepsis/septic shock after a strict resuscitation protocol: a double-blind randomized placebo controlled trial. Crit Care Med 38(1):93-100

14. Depret F, Sitbon A, Soussi S, De Tymowski C, Blet A, Fratani A et al (2018) Intravenous iloprost to recruit the microcirculation in septic shock patients? Intensive Care Med 44(1):121-122

15. De Backer D, Verdant C, Chierego M, Koch M, Gullo A, Vincent JL (2006) Effects of drotrecogin alfa activated on microcirculatory alterations in patients with severe sepsis. Crit Care Med 34(7):1918-1924 